\title{
The Prevalence of Cerebral Microbleeds in HIV-Infected Hemophilia Patients
}

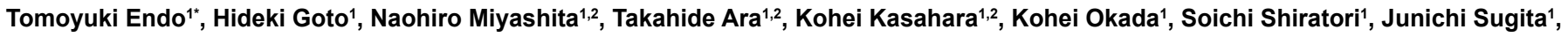
Masahiro Onozawa1, Daigo Hashimoto', Masao Nakagawa ${ }^{1,3}$, Kaoru Kahata', Katsuya Fujimoto', Takeshi Kondo' ${ }^{1}$, Satoshi Hashino ${ }^{1,3}$, Kiyohiro Houkin ${ }^{1}$ and Takanori Teshima ${ }^{1}$

${ }^{1}$ Hokkaido University Hospital, Sapporo, Japan

${ }^{2}$ Japan Foundation for AIDS Prevention, Tokyo, Japan

${ }^{3}$ Hokkaido University Health Care Center, Sapporo, Japan

\section{Abstract}

Objective: Several studies have shown that rates of cerebrovascular events in HIV-infected patients are increased in comparison to uninfected individuals. In addition, cerebral bleeding represents a serious complication in hemophilia patients. Recently, asymptomatic cerebral microbleeds (CMBs), which can be detected by highly sensitive techniques such as $2^{*}$-weighted magnetic resonance imaging (MRI), have emerged as an important marker for predicting symptomatic cerebral bleeding. The aim of the present study was to investigate the prevalence of CMBs in HIVinfected hemophilia patients and to evaluate the association between HIV infection and cerebral bleeding.

Methods: All HIV-infected hemophilia patients (HIV+ HemPts) who visited our hospital from January 2015 to December 2016 were enrolled in this study. In addition, all HIV-uninfected hemophilia patients (HIV- HemPts) who visited our hospital in the same period were enrolled as controls. CMBs were assessed using T2*-weighted MRI. The relationship between cerebral bleeding and the patients' clinical factors was examined.

Results: Two HIV+ HemPts had symptomatic cerebral bleedings during the study period. Twenty-one asymptomatic HIV+ HemPts and $13 \mathrm{HIV}$ - HemPts underwent T2*-weighted MRI. CMBs were observed in $7 \mathrm{HIV}+\mathrm{HemPts}(30.4 \%)$ and $1 \mathrm{HIV}$ - HemPts $(7.7 \%)$. In the multivariate logistic regression analysis, HIV infection was the factor most closely related to cerebral bleeding (odds ratio: 9.78 , p-value: 0.08 ).

Conclusion: This is the first report to investigate the prevalence of CMBs in HIV-infected patients. The prevalence of CMBs or symptomatic cerebral bleeding in HIV+ HemPts was high in comparison to that in HIV- HemPts. The brain screening test by $2^{*}$-weighted MRI seems to be meaningful for HIV+ HemPts.

Keywords: Cerebral microbleeds (CMBs); HIV; Hemophilia; T2*weighted MRI

\section{Introduction}

The development of antiretroviral therapy (ART) has increased the life expectancy of HIV-infected patients [1]. However, they continue to have a higher risk of death than HIV-uninfected individuals. This has been attributed to the increasing incidence of age-related diseases, such as cardiovascular diseases and cerebrovascular diseases. Several studies have shown that rates of cerebrovascular events in HIV-infected patients are increased in comparison to uninfected individuals [2-5]. Although cerebral bleeding is less frequent than cerebral ischemia in HIV-infected patients $[4,6]$, it represents a serious complication, especially in HIVinfected hemophilia patients [7]. Therefore, predicting symptomatic cerebral bleeding is important to avoid complications.

Recent studies have shown that cerebral microbleeds (CMBs), which represent previous asymptomatic microbleeds, can be detected by highly sensitive techniques such as $\mathrm{T} 2^{\star}$-weighted magnetic resonance imaging (MRI) [8-10]. Furthermore, CMBs have emerged as an important marker for predicting symptomatic cerebral bleeding [11].

The aim of the present study was to investigate the prevalence of CMBs in HIV-infected hemophilia patients and to evaluate the association between HIV infection and cerebral bleeding.

\section{Patients and Methods}

\section{Patients}

All HIV-infected adult hemophilia patients (HIV+ HemPts) who visited Hokkaido University Hospital from January 2015 to December 2016 were eligible to enroll in this study. In addition, all HIV-uninfected adult hemophilia patients (HIV- HemPts) who visited in the same period were also enrolled as controls. We excluded patients with metal implants or claustrophobia, which were thought to be contraindications for MRI. This study was performed in accordance with institutional ethical guidelines, including the World Medical Association Declaration of Helsinki and all patients gave their informed consent. For each patient, information was collected on the severity of hemophilia (severe vs mild-moderate), presence of coagulation factor inhibitor, prophylactic use of coagulation factor concentrate, viral infection (HBV or HCV), platelet count and cerebrovascular risk profile (diabetes mellitus, hyperlipidemia, hypertension, smoking habit).

\section{MRI}

CMBs were assessed using $\mathrm{T} 2^{\star}$-weighted MRI, appearing as small

*Corresponding author: Tomoyuki Endo M.D., Ph.D., Department of Hematology, Hokkaido University Hospital, N-15, W-7, Kita-ku, Sapporo, Hokkaido 060-8638, Japan, Tel: 81-11-706-7214; Fax: 81-11-706-7823; E-mail: t-endo@med.hokudai.ac.jp

Received November 20, 2017; Accepted November 25, 2017; Published November 30, 2017

Citation: Endo T, Goto H, Miyashita N, Ara T, Kasahara K, et al. (2017) The Prevalence of Cerebral Microbleeds in HIV-Infected Hemophilia Patients. J AIDS Clin Res 8: 747. doi: 10.4172/2155-6113.1000747

Copyright: (C) 2017 Endo T, et al. This is an open-access article distributed unde the terms of the Creative Commons Attribution License, which permits unrestricted use, distribution, and reproduction in any medium, provided the original author and source are credited. 
Citation: Endo T, Goto H, Miyashita N, Ara T, Kasahara K, et al. (2017) The Prevalence of Cerebral Microbleeds in HIV-Infected Hemophilia Patients. J AIDS Clin Res 8: 747. doi: 10.4172/2155-6113.1000747

Page 2 of 4

dots of signal loss. Cerebral aneurysms were also assessed using magnetic resonance angiography (MRA). All MRI reports were made by experienced radiologist. The relationship between cerebral bleeding and the patients' clinical factors, such as age, the severity of hemophilia, hepatitis virus and HIV infection statuses, hypertension, hyperlipidemia, diabetes mellitus and smoking habit was examined.

\section{MRI parameters}

MRI was performed using a 3.0-T unit (Discovery MR750w 3.0T; GE Health care, Milwaukee, WI, USA). We obtained T2*-weighted MR images of the whole brain using the following parameters: Repetition time (TR) 800 , echo time (TE) 20 , flap angle $30^{\circ}$, slice thickness $5 \mathrm{~mm}$ with a $1.5 \mathrm{~mm}$ interslice gap, field of view (FOV) $240 \times 240 \mathrm{~mm}$ and 352 $\times 192$ matrix. MRA images were obtained using the following parameters: TR 19, TE 3.4 , flap angle $15^{\circ}$, slice thickness $1 \mathrm{~mm}$ without a gap, field of view (FOV) $200 \times 188 \mathrm{~mm}$ and $416 \times 224$ matrix.

\section{Statistical analyses}

Statistical analyses were performed with the JMP software program version 13.0 (SAS Institute, Inc, Cary, NC, USA). Statistical comparisons were made between the HIV+ HemPts and HIV- HemPts groups using the Wilcoxon test for age and Fisher's exact test for other parameters. Univariate and multivariate logistic regression analyses were performed to determine the independent parameters associated with cerebral bleeding. Parameters with $P$-value $<0.5$ in univariate regression analyses were included in multivariate regression analyses. All $P$-values were 2 -sided and a $P$-value of 0.05 was used as the cutoff for statistical significance.

\section{Results}

\section{Patients' characteristics}

Twenty-five HIV+ HemPts and 14 HIV- HemPts visited Hokkaido University Hospital in the study period. One HIV+ HemPt with metal implant, one HIV+ HemPt with claustrophobia and one HIV- HemPt who refused to participate in the study were excluded. Ultimately, $36 \mathrm{pa}-$ tients (23 HIV+ HemPts, 13 HIV- HemPts) were enrolled in the study. The characteristics of each group are listed in Table 1. All patients were male and the median age was 48.0 years old in the HIV+ HemPts group and 47.0 years old in the HIV- HemPts group. The number of patients

\begin{tabular}{|l|c|c|c|}
\hline & $\begin{array}{c}\text { HIV+ HemPts } \\
(\mathbf{n = 2 3})\end{array}$ & $\begin{array}{c}\text { HIV- HemPts } \\
\text { (n=13) }\end{array}$ & P-value \\
\hline Sex (male/female) & $23 / 0$ & $13 / 0$ & 1.00 \\
\hline Age (years), Median (range) & $48.0(36-66)$ & $47.0(25-70)$ & 0.30 \\
\hline Hemophilia & & & \\
\hline A/B & $19 / 4$ & $11 / 2$ & 1.00 \\
\hline Severe, n (\%) & $18(78.3)$ & $8(61.5)$ & 0.44 \\
\hline Inhibitor, n (\%) & $1(4.4)$ & $2(15.4)$ & 0.54 \\
\hline Prophylaxis use, n (\%) & $16(69.6)$ & $4(30.8)$ & 0.04 \\
\hline Hepatitis B, n (\%) & $3(13.0)$ & $2(15.4)$ & 1.00 \\
\hline Hepatitis C, n (\%) & $18(78.3)$ & $6(46.2)$ & 0.07 \\
\hline Thrombocytopenia, n (\%) & $3(13.0)$ & $0(0.0)$ & 0.29 \\
\hline Diabetes mellitus, n (\%) & $4(17.4)$ & $0(0.0)$ & 0.27 \\
\hline Hyperlipidemia, $\mathbf{n}$ (\%) & $4(17.4)$ & $0(0.0)$ & 0.27 \\
\hline Hypertension, n (\%) & $8(34.8)$ & $3(23.1)$ & 0.71 \\
\hline Smoking, n (\%) & $12(52.1)$ & $6(46.2)$ & 1.00 \\
\hline
\end{tabular}

HIV+ HemPts indicates HIV-infected hemophilia patients; HIV- HemPts, HIVuninfected hemophilia patients. Thrombocytopenia was defined as platelet count less than $100 \times 10^{9} / \mathrm{L}$

Table 1: Patients' characteristics. who use coagulation factor concentrate prophylactically was higher in the HIV+ HemPts group $(P=0.04)$, although the severity of hemophilia was not significantly different between the two groups. The prevalence of hepatitis $\mathrm{C}$ tended to be higher in the HIV+ HemPts group $(P=0.07)$.

\section{Brain MRI findings}

Two HIV+ HemPts had symptomatic cerebral bleeding during the study period. CMBs detected by $\mathrm{T} 2{ }^{\star}$-weighted MRI were observed in 7 (30.4\%) of the $23 \mathrm{HIV}+$ HemPts and $1(7.7 \%)$ of the $13 \mathrm{HIV}$ - HemPts (Table 2). Representative images of CMBs are shown in Figure 1. The

\begin{tabular}{|c|c|c|c|}
\hline & $\begin{array}{c}\text { HIV+ HemPts } \\
(\mathbf{n = 2 3})\end{array}$ & $\begin{array}{c}\text { HIV- HemPts } \\
(\mathbf{n = 1 3 )}\end{array}$ & P-value \\
\hline Cerebral bleeding, n (\%) & $9(39.1)$ & $1(7.7)$ & 0.06 \\
\hline Symptomatic, n (\%) & $2(8.7)$ & $0(0.0)$ & \\
\hline Asymptomatic, n (\%) & $7(30.4)$ & $1(7.7)$ & \\
\hline Cerebral aneurysm, n (\%) & $3(13.0)$ & $0(0.0)$ & 0.29 \\
\hline $\begin{array}{c}\text { Cerebral abnormal findings } \\
\text { (bleeding or aneurysm), n (\%) }\end{array}$ & $11(47.8)$ & $1(7.7)$ & 0.03 \\
\hline
\end{tabular}

HIV+ HemPts indicates HIV-infected hemophilia patients; HIV- HemPts, HIVuninfected hemophilia patients.

\section{Table 2: Brain MRI findings.}
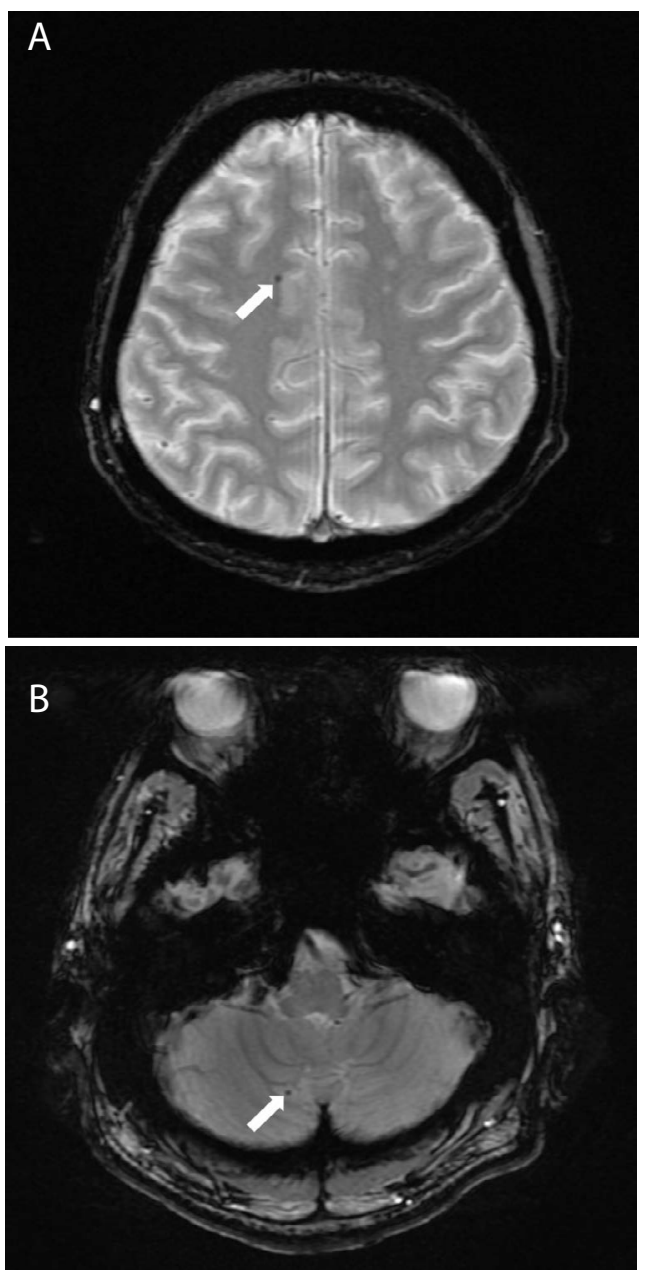

Figure 1: Representative MRI findings in an HIV-infected hemophilia patient. T2* -weighted MRI demonstrating cerebral microbleeds in the right frontal lobe (a), and the right lobe of the cerebellum (b). 


\begin{tabular}{|c|c|c|c|c|}
\hline \multirow[b]{2}{*}{ Clinical factors } & \multicolumn{2}{|c|}{ Univariate analysis } & \multicolumn{2}{|c|}{ Multivariate analysis } \\
\hline & Odds ratio $(95 \% \mathrm{Cl})$ & $P$-value & Odds ratio $(95 \% \mathrm{Cl})$ & $P$-value \\
\hline Age over 50 years & $1.36(0.32-5.89)$ & 0.68 & & \\
\hline Severe hemophilia & $0.86(0.17-4.28)$ & 0.85 & & \\
\hline Inhibitor & $1.33(0.11-16.57)$ & 0.82 & & \\
\hline Hepatitis B & $5.14(0.71-37.15)$ & 0.10 & $7.14(0.50-102.70)$ & 0.15 \\
\hline Hepatitis C & $0.67(0.15-3.03)$ & 0.60 & & \\
\hline HIV & $7.71(0.85-69.98)$ & 0.07 & $23.06(0.84-631.64)$ & 0.06 \\
\hline Thrombocytopenia & $1.33(0.11-16.57)$ & 0.82 & & \\
\hline Diabetes mellitus & $0.00(0.00-)$ & 1.00 & & \\
\hline Hyperlipidemia & $3.00(0.36-24.9)$ & 0.31 & $3.66(0.32-41.71)$ & 0.30 \\
\hline Hypertension & $0.96(0.20-4.72)$ & 0.96 & & \\
\hline Smoking & $0.31(0.07-1.50)$ & 0.15 & $0.27(0.04-1.80)$ & 0.18 \\
\hline
\end{tabular}

Thrombocytopenia was defined as platelet count less than $100 \times 10^{9} / \mathrm{L}$.

Table 3: Clinical factors associated with cerebral bleeding for all patients in this study $(n=36)$.

prevalence of the total cerebral bleeding (symptomatic bleeding or asymptomatic CMBs) tended to be high in the HIV+ HemPts group $(P=0.06)$. Cerebral aneurysms detected by MRA were observed in 3 HIV+ HemPts but in no HIV- HemPts. The number of patients with abnormal findings on brain MRI (cerebral bleedings or aneurysms) was significantly higher in the HIV+ HemPts than in the HIV- HemPts $(P=0.03)$.

\section{Risk factors of cerebral bleedings}

We examined the clinical factors associated with cerebral bleeding for all hemophilia patients in this study (Table 3). In the univariate and the multivariate logistic regression analyses, HIV infection was the factor most closely related to cerebral bleeding, though there was no statistical significance $(\mathrm{P}=0.07$ and 0.08 , respectively).

\section{Discussion}

Several studies have shown that rates of cardiovascular and cerebrovascular events in HIV-infected patients are increased in comparison to uninfected individuals $[2-4,12,13]$. Chow et al. showed that the incidence rate of symptomatic cerebral bleeding was 2.29 per 1,000 person-years in HIV-infected patients compared with 1.23 per 1,000 person-year in uninfected individuals [3].

With the development of MRI techniques, CMBs have emerged as an important maker of cerebral vessel disease. CMBs are detected as small, rounded, homogeneous areas of signal loss on T2*-weighted MRI, representing hemosiderin deposits and they are a result of minor blood leakage through damaged blood vessels [14]. They are not commonly seen on conventional MRI or computed tomography imaging. It has been reported that individuals who have CMBs are 4 to 50 times more likely to experience symptomatic cerebral bleeding than those who do not have CMBs $[11,15]$. Therefore, brain screening examinations by T2*-weighted MRI seem to be beneficial in some disease settings, such as in HIV-infected patients. However, no reports have investigated the prevalence of CMBs in $\mathrm{HIV}$-infected patients so far.

CMBs can be seen even in the normal elderly population. The prevalence is reported to range from $3.1 \%$ to $7.7 \%$ and is associated with advanced age [10,16-18]. Vernooij et al. investigated the prevalence of CMBs in the general population $\geq 60$ years of age and showed that the prevalence of CMBs increased with age, from $17.8 \%$ in those $60-69$ years of age to $38.3 \%$ in those $\geq 80$ years of age [19]. Regarding hemophilia patients, Zanon et al. performed brain MRI in 44 adult patients with hemophilia and CMBs were detected in 3 patients $(6.8 \%)$ [20]. These reports suggest that hemophilia is not associated with the development of CMBs. In our present study, the prevalence of CMBs in HIV+ HemPts was $30.4 \%$. When cases with symptomatic cerebral bleeding were added, the prevalence of cerebral bleeding in HIV+ HemPts was raised to $39.1 \%$. This is extremely high for the age group (median 48.0 years, range 36-66). In contrast, the prevalence of CMBs in HIV- HemPts was only $7.7 \%$, which is roughly the same as in healthy individuals or hemophilia patients.

In this study, HIV infection was the factor most closely related to the development of cerebral bleeding in all hemophilia patients, although there was no statistical significance. Rasmussen et al. reported that injection drug abuse, a low CD4 count and exposure to abacavir were risk factors of cerebrovascular events in HIV-infected patients [4]. In our present study, there were no patients who abused injection drugs. The HIV load was undetectable in 21 patients (91.3\%) and CD4 counts were not suppressed in the patients. Seven patients (30.4\%) used abacavir, but exposure to abacavir was not associated with the presence of CMBs.

Asymptomatic cerebral aneurysms were observed in 3 HIV+ HemPts in this study but in no HIV- HemPts. This finding is compatible with previous reports [21-23]. In those reports, chronic inflammation caused by HIV itself or by opportunistic infections was thought to be responsible for vulnerabilities of blood vessels and related to the formation of cerebral aneurysms. All HIV + HemPts in the present study had a long history of HIV infection, as they had been infected with HIV via blood coagulation factor concentrate in the 1980s. Therefore, long-term micro inflammation due to HIV may cause CMBs or cerebral aneurysms in HIV+ HemPts.

In the present study, the number of patients with abnormal findings on brain MRI (cerebral bleedings or aneurysms) was significantly higher in HIV+ HemPts than in HIV- HemPts $(P=0.03)$. Although the small sample size limited the power of the analysis, these findings indicate that brain screening by MRI is beneficial for HIV+ HemPts. To prevent future symptomatic cerebral bleeding, we managed the blood pressure more strictly, re-evaluated hemophilia treatments and encouraged lifestyle improvements, such as smoking cessation, for patients in whom CMBs were detected by T2*-weighted MRI. Patients in whom cerebral aneurysms were detected by MRA were followed up intensively by a brain surgeon.

\section{Conclusion}

In conclusion, this is the first report to investigate the prevalence of CMBs in HIV-infected patients. The prevalence of CMBs in HIV+ 
Citation: Endo T, Goto H, Miyashita N, Ara T, Kasahara K, et al. (2017) The Prevalence of Cerebral Microbleeds in HIV-Infected Hemophilia Patients. J AIDS Clin Res 8: 747. doi: 10.4172/2155-6113.1000747

HemPts was higher than in HIV- HemPts. The evaluation of CMBs on T2*-weighted MRI may help clinicians tailor their therapeutic decisions in these patents.

\section{Financial Support}

This study was supported in part by a grant-in-aid for scientific research from the Ministry of Health of Japan.

\section{References}

1. Lohse N, Hansen AB, Pedersen G, Kronborg G, Gerstoft J, et al. (2007) Survival of persons with and without HIV infection in Denmark, 1995-2005. Ann Intern Med 146: 87-95.

2. Chow FC, Regan S, Feske S, Meigs JB, Grinspoon SK, et al. (2012) Comparison of ischemic stroke incidence in HIV-infected and non-HIV-infected patients in a US health care system. J Acquir Immune Defic Syndr 60: 351-358.

3. Chow FC, He W, Bacchetti P, Regan S, Feske SK, et al. (2014) Elevated rates of intracerebral hemorrhage in individuals from a US clinical care HIV cohort. Neurology 83: 1705-1711.

4. Rasmussen LD, Engsig FN, Christensen H, Gerstoft J, Kronborg G, et al. (2011) Risk of cerebrovascular events in persons with and without HIV: A Danish nationwide population-based cohort study. AIDS 25: 1637-1646.

5. Cole JW, Pinto AN, Hebel JR, Buchholz DW, Earley CJ, et al. (2004) Acquired immunodeficiency syndrome and the risk of stroke. Stroke 35: 51-56.

6. Pinto AN (1996) AIDS and cerebrovascular disease. Stroke 27: 538-543.

7. Chorba TL, Holman RC, Clarke MJ, Evatt BL (2001) Effects of HIV infection on age and cause of death for persons with hemophilia A in the United States. Am J Hematol 66: 229-240.

8. Chan S, Kartha K, Yoon SS, Desmond DW, Hilal SK (1996) Multifocal hypointense cerebral lesions on gradient-echo MR are associated with chronic hypertension. AJNR Am J Neuroradiol 17: 1821-1827.

9. Roob G, Fazekas F (2000) Magnetic resonance imaging of cerebral microbleeds. Curr Opin Neurol 13: 69-73.

10. Kato H, Izumiyama M, Izumiyama K, Takahashi A, Itoyama $Y(2002)$ Silent cerebral microbleeds on T2*-weighted MRI: Correlation with stroke subtype, stroke recurrence, and leukoaraiosis. Stroke 33: 1536-1540.

11. Imaizumi T, Horita Y, Hashimoto Y, Niwa J (2004) Dotlike hemosiderin spots on
T2*-weighted magnetic resonance imaging as a predictor of stroke recurrence: A prospective study. J Neurosurg 101: 915-920.

12. Islam FM, Wu J, Jansson J, Wilson DP (2012) Relative risk of cardiovascular disease among people living with HIV: A systematic review and meta-analysis. HIV Med 13: 453-468.

13. Lang S, Mary-Krause M, Cotte L, Gilquin J, Partisani M, et al. (2010) Increased risk of myocardial infarction in HIV-infected patients in France, relative to the general population. AIDS 24: 1228-1230.

14. Fazekas F, Kleinert R, Roob G, Kleinert G, Kapeller P, et al. (1999) Histopathologic analysis of foci of signal loss on gradient-echo T2*-weighted MR images in patients with spontaneous intracerebral hemorrhage: evidence of microangiopathy-related microbleeds. AJNR Am J Neuroradiol 20: 637-642.

15. Bokura H, Saika R, Yamaguchi T, Nagai A, Oguro H, et al. (2011) Microbleeds are associated with subsequent hemorrhagic and ischemic stroke in healthy elderly individuals. Stroke 42: 1867-1871.

16. Roob G, Schmidt R, Kapeller P, Lechner A, Hartung HP, et al. (1999) MRI evidence of past cerebral microbleeds in a healthy elderly population. Neurology 52: 991-994.

17. Kinoshita T, Okudera T, Tamura H, Ogawa T, Hatazawa J (2000) Assessment of lacunar hemorrhage associated with hypertensive stroke by echo-planar gradient-echo T2*-weighted MRI. Stroke 31: 1646-1650.

18. Tsushima Y, Tanizaki Y, Aoki J, Endo K (2002) MR detection of microhemorrhages in neurologically healthy adults. Neuroradiology 44: 31-36.

19. Vernooij MW, van der Lugt A, Ikram MA, Wielopolski PA, Niessen WJ, et al (2008) Prevalence and risk factors of cerebral microbleeds: the Rotterdam Scan Study. Neurology 70: 1208-1214.

20. Zanon E, Manara R, Milan M, Brandolin B, Mapelli D, et al. (2014) Cognitive dysfunctions and cerebral microbleeds in adult patients with haemophilia A: A clinical and MRI pilot-study. Thromb Res 134: 851-855.

21. Pagiola IC, Paiva AL, de Aguiar GB, de Oliveira AC, Conti ML, et al. (2016) Cerebral aneurysms associated with human immunodeficiency virus in adults: Literature review and new perspectives. Rev Assoc Med Bras 62: 85-89.

22. Goldstein DA, Timpone J, Cupps TR (2010) HIV-associated intracranial aneurysmal vasculopathy in adults. J Rheumatol 37: 226-233.

23. Statler JD, Slaughter CR, Ronsivalle JA (2007) Human immunodeficiency virus arteriopathy of the adult cerebral circulation. Mil Med 172: 647-649. 